\title{
Clinical guideline: definition and classification of epilepsy
}

\author{
Juan C. Reséndiz-Aparicio ${ }^{1}$, Juan C. Pérez-García², Efraín Olivas-Peña ${ }^{3}$,Enrique García-Cuevas ${ }^{4}$, Yuridia \\ L. Roque-Villavicencio ${ }^{5}$, Marisela Hernández-Hernández ${ }^{6}$, Jaime I. Castro-Macías ${ }^{7}$ and \\ Jesús D. Rayo-Mares ${ }^{8}$ \\ ${ }^{1}$ PPE, Instituto Nacional de Neurología y Neurocirugía Dr. Manuel Velasco Suárez y Hospital Psiquiátrico Infantil Dr. Juan N. Navarro, Mexico City; \\ ${ }^{2}$ Hospital Christus Muguerza UPAEP, Puebla; ${ }^{3} \mathrm{Hospital}$ de la Mujer, Yautepec, Morelos; ${ }^{4} \mathrm{Hospital}$ General Acapulco, Guerrero; ${ }^{5} \mathrm{Hospital}$ Civil de \\ Guadalajara, Jalisco; ${ }^{6}$ Hospital Central Sur de Alta Especialidad Pemex, Mexico City; ${ }^{7}$ Hospital Regional de Alta Especialidad del Bajío, León, \\ Guanajuato; ${ }^{8}$ Hospital de Pediatría del Centro Médico Nacional Siglo XXI IMSS, Mexico City. Mexico
}

\begin{abstract}
The current definition of epilepsy proposes three possibilities to consider this diagnosis, the first when a patient has two or more unprovoked or reflex seizures in $>24 \mathrm{~h}$; the second, an unprovoked or reflex seizure with at least a $60 \%$ probability of continuing to present seizures; and the third, the presence of an epileptic syndrome. The classification of the type of seizure divides them into three possibilities depending on how they begin; they can be of focal, generalized, or unknown onset. Focal seizures can be subclassified into those that have or have not lost consciousness, then categorized as to whether the symptoms are motor or non-motor, and further give a descriptor of the event, which is nothing else but the description of symptoms and signs presented by the patient during his seizure. The classification of the type of epilepsy, proposes three diagnostic levels, the first related to the type of seizure, the second to the type of epilepsy, and the third to the type of epileptic syndrome, without forgetting etiology and comorbidity. These concepts are basic for the approach of any patient who presents epilepsy.
\end{abstract}

Key words: Epilepsy. Definition. Classification.

\section{Introduction}

This being a clinical guide, it is developed based on research questions under the PICO method; answers are presented, and it concludes with recommendations. In this clinical guide, we do not mention levels of evidence since the references used are current articles from the International League Against Epilepsy (ILAE) ${ }^{1-3}$, which is the official agency for this disease worldwide, and not a review of the available evidence. The ILAE and its workgroups have strived to provide a standard terminology to be used worldwide.

\section{Question 1. What is an epileptic seizure?}

An epileptic seizure is defined as the transitory manifestation of signs and/or symptoms caused by an anomalous excessive neuronal activity in the brain ${ }^{4}$.

\section{Correspondence:}

Juan Carlos Reséndiz-Aparicio

E-mail: jc_doc@yahoo.com
Disponible en internet: 12-04-2019 Rev Mex Neuroci. 2019;20(2):63-68

www.revmexneurociencia.com 1665-5044/@ 2019. Academia Mexicana de Neurología A.C. Published by Permanyer México. This is an open access article under the CC BY-NC-ND license (http://creativecommons.org/licenses/by-nc-nd/4.0/). 


\section{Question 2. What is an unprovoked seizure?}

The term unprovoked implies the absence of a temporal or reversible factor that reduces the convulsive threshold and provokes a seizure at that moment. The opposite of this type of phenomenon is an acute symptomatic seizure, defined as a seizure that occurs in temporal relation during cerebral injury, which can be metabolic, infectious, toxic, structural, or inflammatory ${ }^{4,5}$.

\section{Question 3. What is the current definition of epilepsy?}

A diagnosis of epilepsy is established after any of the following situations ${ }^{4}$ :

1. Two or more unprovoked or reflex seizures occurring with $>24 \mathrm{~h}$ difference.

2. An unprovoked or reflex seizure with a probability of at least $60 \%$ of presenting a future seizure (similar range as the general risk of recurrence, after two unprovoked seizures that appear in the following 10 years).

3. Diagnosed epileptic syndrome.

\section{Question 4. How is the risk of recurrence of an epileptic seizure defined?}

The events that have been documented to increase the risk of recurrence of an epileptic seizure are cerebrovascular disease, both ischemic and hemorrhagic, cranioencephalic trauma, abnormal electric activity in an EEG compatible with an epileptic seizure, or a cranial magnetic resonance showing a lesion compatible with a diagnosis of epilepsy ${ }^{5}$. If a patient, after a first unprovoked seizure is predisposed to continued generation of seizures of at least $60 \%$, a diagnosis of epilepsy must be considered ${ }^{4}$. If this is not the case, to establish a diagnosis of epilepsy one must consider option one (two or more unprovoked seizures $>24 \mathrm{~h}$ apart).

Epilepsy is considered as resolved in those patients that have an age-dependent epileptic syndrome but have grown older than the corresponding age for the specific syndrome or in patients that have remained seizure-free for the past ten, without taking antiepileptic drugs in the last 5 years ${ }^{4}$.

\section{Question 5. What is the current classification by type of epileptic seizure?}

The ILAE decided to modify the classification system for epileptic seizures in $1981^{6}$, and update it in $2010^{7}$.
The classification is divided into three, depending on the patient's symptoms at seizure onset:

Focal onset seizure/Generalized onset seizure/Unknown onset seizure.

Focal onset seizures originate within a network limited to one hemisphere; they can be localized or more widely distributed. Generalized seizures are those that originate at one point with wide and rapid participation of bilaterally distributed networks. Those seizures where it cannot be distinguished whether they are of focal or generalized onset with a confidence level of $80 \%$, must be considered of unknown onset (Fig. 1).

\section{Question 6. How are focal onset epileptic seizures currently classified?}

The state of awareness is a differentiating factor for the type of seizure in focal seizures ${ }^{1,2}$. Awareness refers to the relationship between oneself and the external environment. In epilepsy, we establish awareness as the mental state, with both subjective and objective aspects, that encompasses the sense of self as a unique entity, capable of response, and memory. A focal seizure is classified by the degree of awareness during onset, which can be altered at any time during the event.

Focal seizures, with or without impaired awareness, can be subclassified depending on whether they initiate with motor or non-motor symptoms. In addition, the 2017 classification recommends adding what they call "descriptors," which are symptoms and signs that the patient presents during the seizure ${ }^{1,2}$. Descriptors have been divided into six groups: motor, automatisms, sensory, emotional, cognitive, or autonomic. To remember these descriptors more easily, you can use the mnemotechny MASECA, which is the most famous brand of tortilla dough in Mexico ${ }^{8}$.

\section{Question 7. How are the symptoms and signs divided into focal seizures?}

\section{Motor}

Involvement of the musculature in any way. An increase (positive) or decrease (negative) in muscular contraction to produce movement. The most frequent are: motor arrest, astatic, clonic, dysarthria, dystonic, pelvic thrusting, figure-of-4, hypokinetic, hyperkinetic, incoordination, Jacksonian, myoclonic, paralysis, paresis, pedaling, fencer's posture, and versive.

The most frequent clinical confusion is in differentiating clonic seizures from myoclonic seizures. A clonic seizure is a repetitive, regular movement that can be 


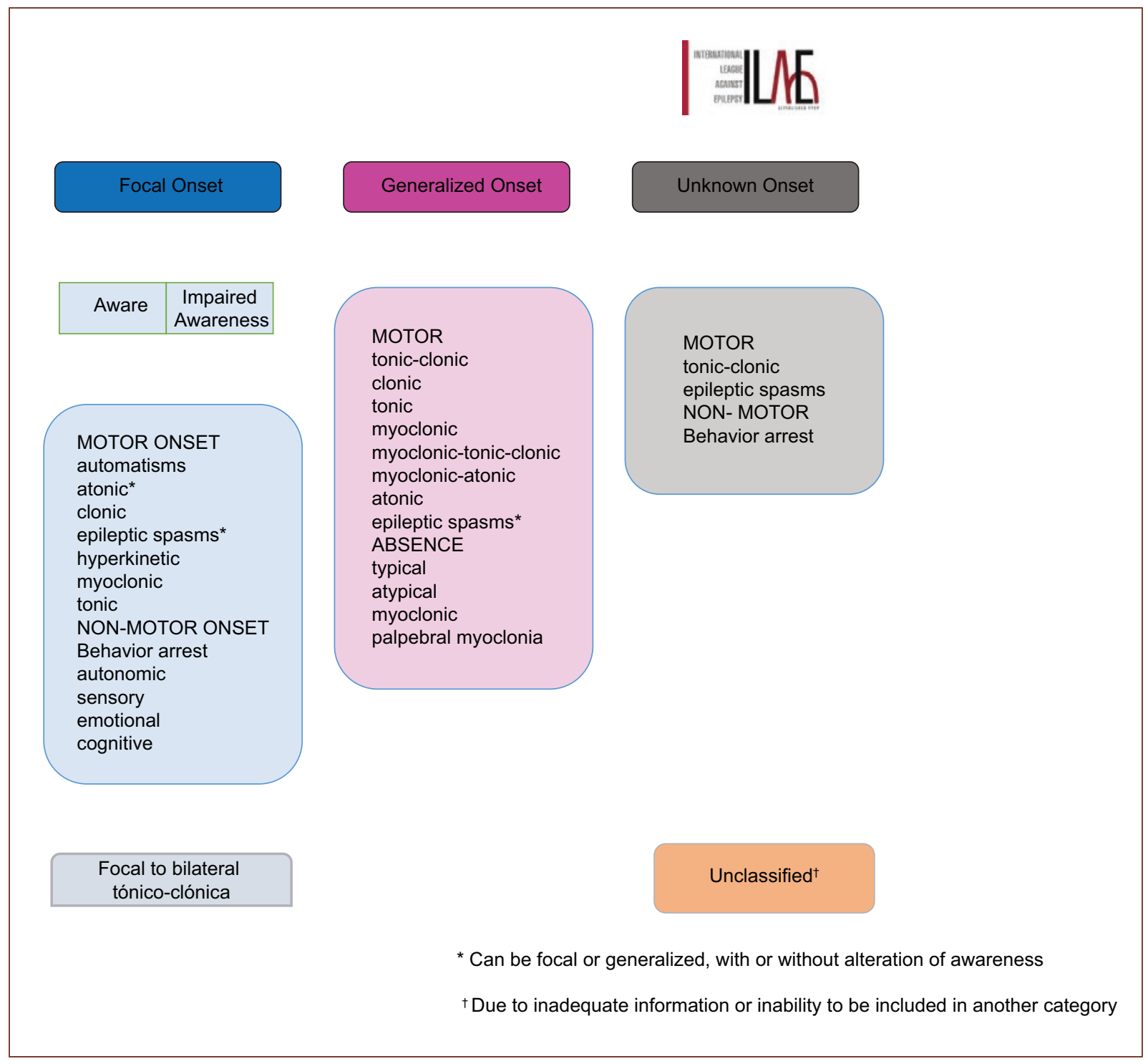

Figure 1. Classification by type of seizure. ILAE, 2017.

symmetrical or asymmetrical and that involves the same muscle groups. A myoclonic seizure is a brief ( $<100 \mathrm{~ms})$, sudden, involuntary, single or multiple contractions of the muscles or muscle groups, with variable topography (axial, proximal, or distal extremities). The myoclonic seizure is less repetitive and sustained.

\section{Automatisms}

Motor activity, which is more or less coordinated, that generally occurs when awareness is deteriorated and after which the subject is generally (but not always) amnesiac. It commonly resembles a voluntary movement. The most frequent include: aggression, manual, oral-facial, perseveration, sexual, undressing, vocalization, walking, or running.

\section{Sensory}

Related with the senses, thus, in this case, are not signs but symptoms. The most frequently reported symptoms are: auditory, gustatory, olfactory, somatosensory, vestibular, visual, and pain.

\section{Emotional}

These are seizures that present as having an emotion as a prominent initial feature, such as fear, pleasure or 
spontaneous euphoria, laughter, crying, expressed in bursts, or flares.

\section{Cognitive}

The most frequently reported data from this group are: acalculia, aphasia, attention impairment, déjà vu or jamais vu phenomena, dysphasia, illusions, memory impairment, forced thinking, altered responsiveness, or hallucinations.

\section{Autonomic}

Alteration of the autonomic nervous system function, which may involve the cardiovascular system, pupils, diaphoresis, the gastrointestinal tract, vasomotor and thermoregulation functions.

In focal seizures, there are two sections that were not included in previous classifications, the epileptic spasm (previously only classified within generalized seizures) and behavior arrest ${ }^{1,2}$.

A focal onset seizure, with or without impaired awareness, motor or non-motor, can progress to a bilateral tonic-clonic activity. The term previously used for this type of seizure was a secondary generalized partial seizure. At present, we must classify it as a focal onset seizure that evolves into a bilateral tonic-clonic seizure.

The term bilateral tonic-clonic is used for focal seizures that propagate to both cerebral hemispheres, while generalized is for seizures that originate simultaneously in both cerebral hemispheres.

\section{Question 8. ¿How are generalized epileptic seizures currently classified?}

They are divided into seizures with motor or non-motor symptoms. Among those with motor, symptoms are the generalized tonic-clonic, clonic, tonic, myoclonic, myoclonic-tonic-clonic, myoclonic-atonic seizures, and the epileptic spasm. For non-motor, there are absence seizures ${ }^{1,2}$. A myoclonic-tonic-clonic is a type of generalized seizure that was not included in the previous classification. These seizures imply one or various bilateral jerks (myoclonic) of the extremities, followed by a tonic-clonic seizure ${ }^{1,2,8}$. Atonic seizures present sudden loss or reduction in muscle tone involving musculature of the head, trunk, mandible, or extremities.

The epileptic spasm is usually more sustained than a myoclonic movement, but not as sustained as a tonic seizure. It frequently occurs in clusters or bursts. Epileptic spasms are more frequent in children but can occur at all ages.

Absence seizures can be divided into four types: Typical absence, atypical absence, myoclonic absence, and absence with palpebral myoclonus. Typical absence seizures are of sudden onset, interrupting ongoing activities, blank stare, does not respond when spoken to, lasting from seconds to half a minute, and with very quick recovery. It's important to remember that the word "absence" is not synonymous with blank stare since this can also be encountered in focal onset seizures ${ }^{2}$.

Atypical absence seizures show changes in tone that are more pronounced than in typical absence; the onset and cessation are not abrupt. Myoclonic absence presents with sudden, brief ( $<100 \mathrm{~ms})$, involuntary, non-repetitive nor sustained, and absence. In absence with palpebral myoclonus, we observe eyelids jerking at a frequency under 3 per second, eyes commonly deviated upward, generally lasting $<10 \mathrm{~s}$, frequently precipitated by ocular closure, with high possibility of photosensitivity.

\section{Question 9. When do we classify an epileptic seizure under the heading of unknown onset or unclassified?}

When there is no evidence about the onset of the seizure, focal or generalized, it may be classified under the heading of unknown onset seizure, and in this case, a limited classification can be carried out with the findings that were observed.

The heading "Unclassified" applies to the type of seizure when there are no data described in the ILAE classification of 2017, either because the information is inadequate or because of unusual characteristics ${ }^{1,2}$.

\section{Question 10. How are epilepsy and epileptic syndromes classified?}

Since April 2017 the epilepsy classification set forth by the ILAE establishes three levels of diagnosis: the first level is the type of seizure, which incorporates all of the concepts in all diagnostic levels revised previously, focal, generalized or of unknown onset, and now we have added two important concepts at all diagnostic levels: patient comorbidities (associated pathological entities) and etiology. Some patients can only stay at this diagnostic level because it is not possible to study more, and this can be valid or be the 


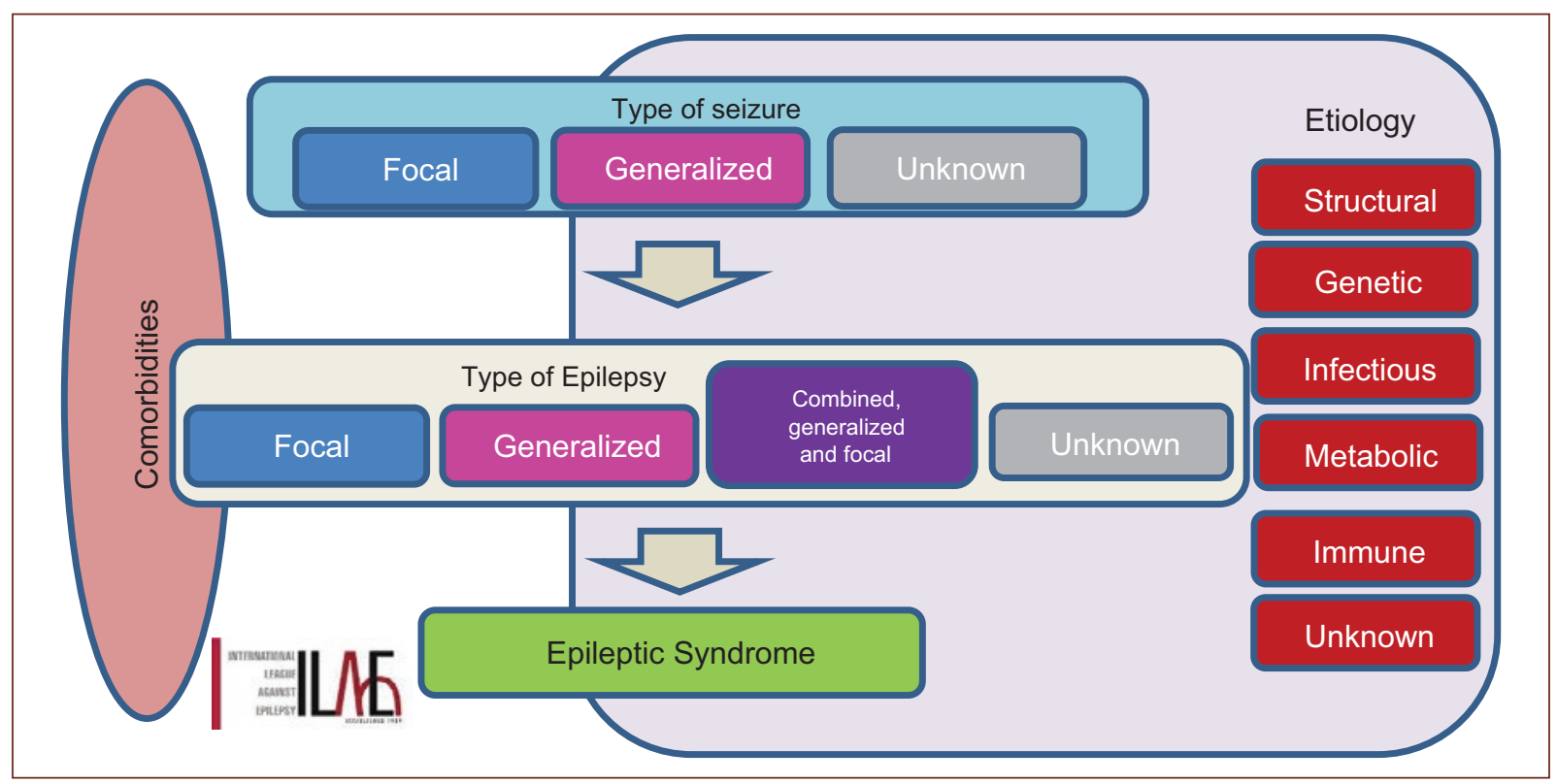

Figure 2. Classification of Epilepsy. ILAE 2017.

first step in their evaluation. The second diagnostic level applies when there is at least one encephalogram and a cerebral imaging study. At this level one must establish the type of epilepsy, which can be focal, generalized, or combined; this is for focal, generalized (which is common in various epileptic syndromes) or unknown type seizures. The etiologic diagnosis can be in any of the following fields: structural, genetic, infectious, metabolic, immune, or unknown. On occasion there can be $>1$ etiology in the same patient, for example, a patient with tuberous sclerosis that has cortical tubers would suggest a structural etiology, but also genetic due to the base disease. The third diagnostic level constitutes an epileptic syndrome. A group of characteristics that incorporates types of seizure, specific EEG findings, characteristics form imaging studies, age-dependent frequency, age at onset, and remission when applicable, specific triggers, variations during the day, on occasion prognosis, distinctive comorbidities both intellectual and psychiatric, and all can have implications on etiology and treatment. This classification eliminates the term benign which has been substituted by the terms autolimited or drug responsive (Fig. 2).

Epileptic syndromes can be classified by age groups, as was established by the ILEA classification of $2010^{7}$, but that is subject for another Guide.

\section{Question 11. How are seizures and epilepsy in the newborn classified?}

There are various classifications for neonatal seizures. The Volpe classification ${ }^{9}$ considers the clinical findings (mostly used by pediatricians and neonatologists) and the Mizrahi classification ${ }^{10}$ considers the physiopathologic origin, be it epileptic or non-epileptic. In 2018, the ILAE issued a new proposal to classify seizures in the newborn (Fig. 3) that includes four parts: the presentation, established in the critically ill newborn who must be under vigilance in view of the possibility of a seizure; diagnosis, by performing a video-EEG; manifestation, which could be by clinical signs or only electroencephalographic data; and fourth, the type of seizure, which could be with motor symptoms. It is called sequential when the symptoms in the newborn show a motor sequence and the seizure is of a non-motor type.

\section{\begin{tabular}{l|l} 
Recommendation & Level of recommendation
\end{tabular}}

It's desirable for health R-PPE

professionals to know the

current definition of epilepsy to

be able to apply it

It's desirable that health R-PPE professionals know and apply the current classification of the types of epileptic seizures and epilepsies 


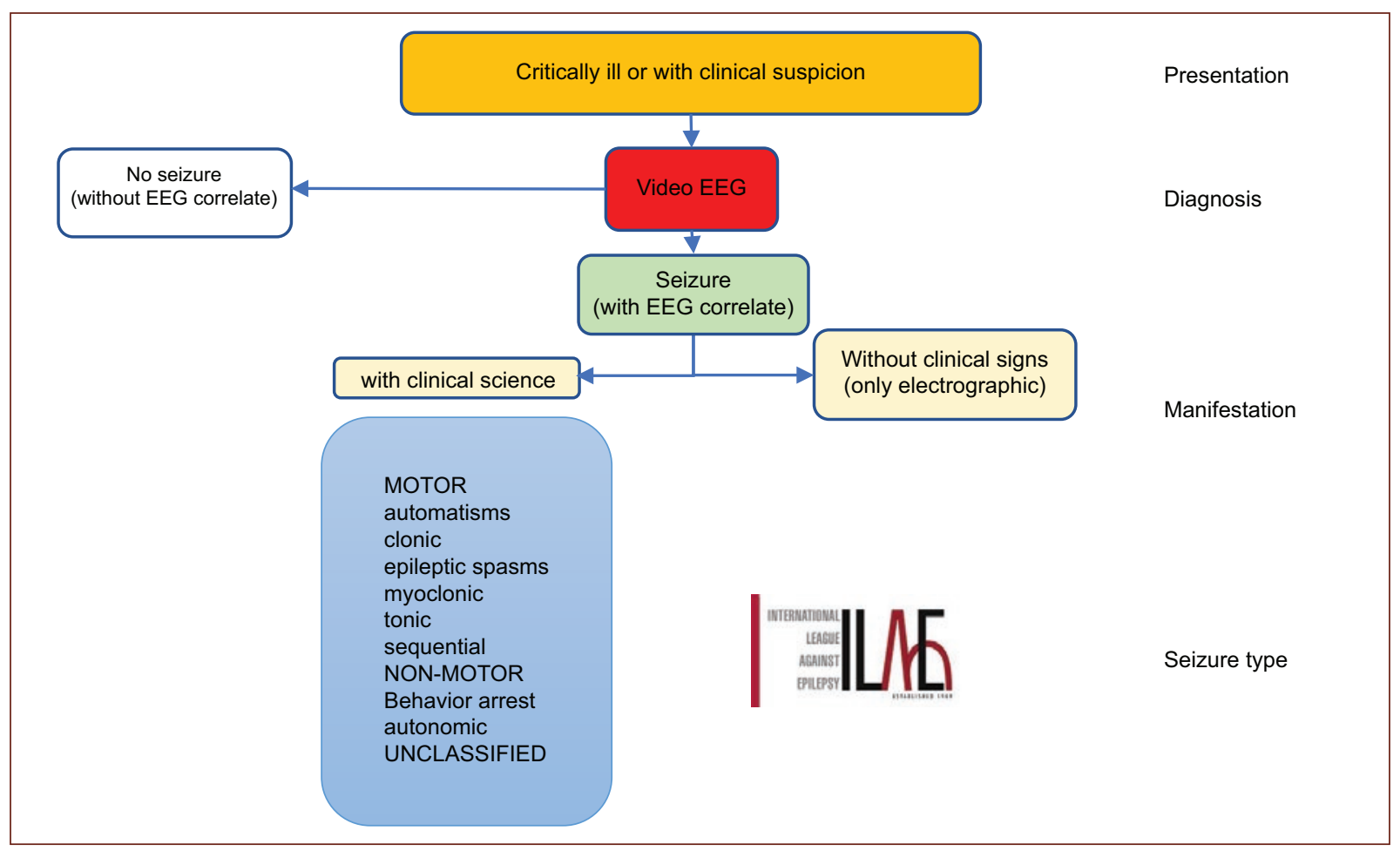

Figure 3. Diagram of the proposed classification for seizures in the newborn.

\section{Conflicts of interest}

In this article, there are no conflicts of interest by the authors or the source of funding.

\section{References}

1. Fisher RS, Cross JH, French JA, et al. Operational classification of seizure types by the international league against epilepsy: position paper of the ILAE commission for classification and terminology. Epilepsia. 2017:58:522-30

2. Fisher RS, Cross JH, D'Souza $C$, et al. Instruction manual for the ILAE 2017 operational classification of seizure types. Epilepsia. 2017;58:531-42.

3. Scheffer IE, Berkovic S, Capovilla G, et al. ILAE classification of the epilepsies: position paper of the ILAE commission for classification and terminology. Epilepsia. 2017;58:512-21.
4. Fisher R, Acevedo C, Arzimanoglou A, et al. Definición clínica practica de la Epilepsia. Epilepsia. 2014;55:475-82.

5. Thurman DJ, Beghi E, Begley CE, et al. Standards for epidemiologic studies and surveillance of epilepsy. Epilepsia. 2011;52 Suppl 7:2-6.

6. Bancaud J, Rubio-Donnadieu F, Seino M, Dreifuss F, Penry K. Proposal for revised clinical and electroencephalographic classification of epileptic seizures. From the commission on classification and terminology of the international league against epilepsy. Epilepsia. 1981;22:489-501.

7. Berg AT, Berkovic SF, Brodie MJ, et al. Revised terminology and concepts for organization of seizures and epilepsies: report of the ILAE commission on classification and terminology, 2005-2009. Epilepsia. 2010:51:676-85.

8. Rubio F, Resendiz JC, Alonso MA, Senties H. Epilepsia. $1^{\text {st }}$ ed. México: Editorial Alfil; 2016. p. 27-42.

9. Volpe JJ. Neonatal seizures: current concepts and revised classification. Pediatrics. 1989;84:422-8.

10. Mizrahi EM, Kellaway P. Characterization and classification of neonata seizures. Neurology. 1987;37:1837-44. 